\title{
Patients' comorbidities reduce the clinical value of emergency colonoscopy: results of a retrospective cohort study
}

\section{(๑) $\odot \ominus$}

\section{Authors}

Cristiano Cremone ${ }^{1,5}$, Anouk Esch², Charlotte Gagniere ${ }^{2}$, Alessandro Fugazza², Faria Mesli², Michael Levy², Aurelien Amiot $^{2}$, Alexis Laurent ${ }^{3}$, Yann LeBaleur ${ }^{2}$, Francois Hemery ${ }^{4}$, Nicolas De'Angelis ${ }^{3}$, Francesco Brunetti ${ }^{3}$, Iradj Sobhani ${ }^{1,2}$

Institutions

1 EC2M3: Department of Academic Research (EA7375) Université Paris Est Créteil (UPEC) - Val de Marne, France

2 Department of Liver and Gastroenterology, Hôpital Henri Mondor - Assistance Pulique Hôpitaux de Paris (APHP), Paris, France

3 Department of Liver and GI surgery, Hôpital Henri Mondor - Assistance Pulique Hôpitaux de Paris (APHP), Paris, France

4 Department of Bio Informatic, Hôpital Henri Mondor Assistance Pulique Hôpitaux de Paris (APHP), Paris, France

5 Department of Advanced Biomedical Sciences, University Federico II of Naples, Naples, Italy

submitted 6.12 .2016

accepted after revision 22.5.2017

\section{Bibliography}

DOI https://doi.org/10.1055/s-0043-118001 | Endoscopy International Open 2017; 05: E1119-E1127

(c) Georg Thieme Verlag KG Stuttgart · New York

ISSN 2364-3722

Corresponding author

Iradj Sobhani, Professor, MD, PhD, Department of Gastroenterology, Henri Mondor Hospital and University Paris-Est CRETEIL (UPEC), Dir. Of EA-7375 Cancer Research Lab. 51, avenue du Mal de Lattre de Tassigny - 94010 CRETEIL - France

Fax: 33-1-49 812352

iradj.sobhani@aphp.fr

\section{ABSTRACT}

Background and study aims Urgent endoscopy is often used to diagnose and sometimes treat acute upper gastrointestinal syndromes (hemorrhage, toxic ingestion, and occlusion). However, its suitability concerning the management of lower gastrointestinal conditions in emergency circumstances is controversial.
Patients and methods We studied the role of emergency colonoscopy in diagnosis and treatment of all consecutive patients presenting with acute lower gastrointestinal symptoms referred to our hospital on an emergency basis. All patients were first managed by physicians from the emergency room and/or the intensive care unit (ICU); the treatments included fluid resuscitation, blood transfusion, and antibiotic or cardiotonic as needed. Bowel cleansing was performed to purge the colon of clots, stool, and blood when clinically possible; alternatively, a bowel enema was used. Patients only underwent a computed tomography (CT) scan prior to the colonoscopy in clinically relevant situations. Colonoscopy was performed within 6-36 hours after hospitalization or the beginning of the clinical symptoms (hemorrhage, sepsis, colon distension) or occlusion, as assessed by abdominal CT scan.

Results From 2010 to 2015, 603 patients underwent urgent colonoscopy; among them, 214 (36\%) presented with lower GI bleeding, while 264 (44\%) had symptoms suggestive of intestinal ischemia; almost half $(49 \%, n=295)$ of the patients were hospitalized in the ICU. Patients received therapies, such as clips (15\%), epinephrine injections (5\%), bipolar coagulation (7\%), or devolvulation (3\%) using colonoscopy or antibiotic therapy when needed. No perforation was observed after colonoscopy and only three cases of hemorrhage recurrence were documented as complications after the procedure. Overall, 192 patients died within 1 month after colonoscopy due to four independent risk situations, as follows: septic shock, heart transplantation, multiorgan failure, and ischemic colitis. Only 67 (35\%) underwent urgent intestinal surgery when ischemic colitis was identified, and this did not have a significant effect on the mortality rate.

Conclusions Urgent bedside colonoscopy is feasible and safe for routine use. The highest advantage was observed in patients with red blood hemorrhage, diarrhea, and colon distension when symptoms were not associated with multiorgane failure, heart transplantation, or septic shock. As revealed by colonoscopy and pathological features, ischemic colitis is associated with a bad prognosis, and patients experience a higher rate of early mortality regardless of whether they undergo urgent colon surgery. 


\section{Introduction}

In practice, gastroenterologists are frequently invited to perform urgent colonoscopy, often in critically ill patients. Although hemorrhage remains the main medical problem to be assessed, some other issues, such as sepsis, diarrhea, suspicion of colonic ischemia, and volvulus, are major situations leading to this procedure. Overall, the benefits of urgent colonoscopy for acute abdominal symptoms, including all consecutive issues, have not been investigated so far [1-7]. Moreover, for lower gastrointestinal bleeding (LGIB) the clinical setting is distinct from that relating to upper gastrointestinal bleeding, because different aims and techniques are involved in these disorders. Longitudinal studies have indicated that incidence of hospitalization for lower Gl complications (primarily bleeding) increased by $65 \%$ over a decade, shifting from 20 out of 100,000 persons in 1996 to 33 out of 100,000 persons in 2005 [4]. An alternative reason for urgent colonoscopy is severe sepsis with a gastrointestinal septic and/or ischemic location; in these patients, who have an extremely high risk of recurrent or continued bleeding and multivisceral failure, early diagnosis and the potential application of therapy may be the highest priorities. In some such patients, need for surgery may affect morbidity and mortality [5]. Thus, urgent colonoscopy represents a serious, increasingly important issue for both patients and gastroenterologists. In most cases, however, this procedure is requested in patients with one or numerous comorbidities, making it difficult to best evaluate the safety, as well as the cost-effectiveness, of early colonoscopy [6].

Early colonoscopy is recommended for patients with severe LGIB, although the results in the literature remain contradictory regarding improved outcomes. Prospective controlled studies have reported an extremely small benefit of early colonoscopy and no significant difference between procedures performed within 24 hours after patient admission and those performed at 24 to 72 hours; however, most of these studies have lacked statistical power due to the inclusion of a small number of patients [1]. A recent meta-analysis including 6 of the main available retrospective studies compared the results of 422 patients in the early group and 479 in the delayed group. The 2 groups were comparable in terms of age, hemoglobin on admission, and packed red blood cell transfusion, as well as the rates of in-hospital mortality, re-bleeding, and need for surgery. Consequently, the authors concluded that early colonoscopy-although associated with greater detection of the bleeding source and endoscopic intervention - did not seem necessary [2], despite the positive results of a randomized study [3]. In addition, the guideline for urgent colonoscopy remains ambiguous regarding which patients should benefit from this procedure.

The aim of this study was to evaluate the role of bedside colonoscopy in the management of patients presenting with urgent lower gastrointestinal symptoms and assess how it affects early mortality. We show that patients' comorbidities can significantly affect survival independently of the results of the exam.

\section{Patients and methods}

In our healthcare organization, every exam or test regarding a patient's management is recorded. This is done to evaluate the activity of the health unit. Thus, the reference number of each procedure and the related cause are identifiable; consequently, the incidence and prevalence of pathologies in selected populations regarding the total activity of the unit can be evaluated. This is supported by a software system used to code and collect patient data. The recording system is checked by the Département d'Information Médicale/Department of medical information (DIM). We used data from all recorded consecutive colonoscopies conducted between January 1, 2010 and December 31, 2014 at Mondor Hospital (Créteil, France). To further ensure that no cases were missed, we also searched for patients with diagnostic reference codes that are usually eligible for urgent procedures, for example, rectal bleeding; intestinal angiodysplasia with hemorrhage; blood in stool; melena; pseudomembranous colitis; ulcerative, ischemic, or intestinal pseudo-obstruction/Ogilvie syndrome; megacolon/toxic megacolon; diverticulitis/diverticulosis; enterocolitis; necrotizing gastroenterocolitis; inflammatory bowel disease (IBD); Crohn's disease; vascular abdominal angina; mesenteric ischemia; angiodysplasia; and volvulus.

A total of 6,805 colposcopies were identified; no missing procedures were evident that could constitute a bias. The notes for each patient were reviewed, and every colonoscopy was classified as "urgent" or "not urgent." All those marked "urgent" were included in the study. A colonoscopy was defined as "urgent colonoscopy" when an exam was performed within 36 hours after demand. The choice to extend this period to a 36-hour delay was made because urgent colonoscopy could be scheduled within 24 hours but performed after 24 hours (from the time of request) for a care reason (e. g., patient cardiovascular instability, inadequate bowel preparation), an organizational constraint (procedure could be performed only between 8:00 am and 6:00 pm), lack of availability of materials, and so on. Furthermore, although the large majority of patients had urgent colonoscopies in less than 24 hours, this 6-hour extension of the timing could ensure that all relevant patients who needed urgent colonoscopy were identified.

Authorization for this research was granted by the French National Commission on Liberty and Digital Data (Commission Nationale de l'Informatique et des Libertés). Over 1 year, the following data were retrospectively collected from patients' files: indication for the exam, medical background, and potential colonoscopy-related complications. Follow up was determined using medical records for those patients who had not been discharged within 1 month after the last endoscopic exploration. For all remaining patients, a medical examination was performed 1 to 3 months after the last endoscopy. Information relating to patient status at 72 hours after colonoscopy was available for all patients, since none were discharged prior to this time; this was obtained using the hospitalization report and computerized dossier, where all major events and the patient trajectory are recorded. We considered the following characteristics: need for further colonoscopies, need for sur- 
gery, type of surgery, histology, and mortality. The median follow-up time in the series was 5.12 months, although for death events, the duration was limited to 1 month following the last colonoscopy performed. We also recorded all data from exams, including type and quality of bowel preparation, percentage of cecal intubation, endoscopic findings, and potential endoscopic therapy procedures.

Quality of preparation could be subjective; this was defined by the endoscopist during the procedure and routinely recorded in the results. It was defined as "good" when it allowed the endoscopist to fully perform the exam, correctly examine the source of the problem, and eventually perform an endoscopic hemostatic procedure when needed. The Boston classification could not be used because of the low rate of complete colonoscopies in this urgent context, as well as because the Boston score was not routinely registered at the beginning of the study period due to the failure of scoring at that time. A colonoscopy was defined as "complete" when the cecum was intubated. In contrast, it was defined as "contributive" when colonoscopy affected the precision of diagnosis or contributed to the decision to administer therapy. This included all pathological features colonoscopy could reveal, as well as any related therapy decision mentioned in the medical file during 1 month of follow up if it was directly related to the colonoscopy and/or colonic pathology results. In this regard, among polyps found during the colonoscopy, only those considered as the cause of bleeding or sepsis could cause the colonoscopy exam to be labelled "contributive."

The study site was a large, tertiary-care, academic hospital in a major city. The gastroenterology team specialized in both diagnosis and interventional endoscopy procedures. Mondor Hospital includes five intensive care units (ICUs), as follows: a medical ICU, surgical ICU, specific GI and liver ICU, cardiac ICU, and neurological ICU. The hospital is a reference center for vascular and aortic surgery.

When a patient was hospitalized in 1 of the 5 ICUs of the hospital, urgent colonoscopy was requested by the ICU physicians and performed by a senior endoscopist after evaluation of the patient by the gastroenterology team for urgent processing. In these cases, a cart containing a light source, processor, and colonoscopes was brought to the bedside. In patients not admitted to the ICU, urgent colonoscopies were performed in the endoscopic unit. For patients under sedation and ventilation monitoring or those unable to drink (because of ileus, potentially serious colitis, or organic obstruction), preparation by Normacol enema was performed a few hours before the procedure. In the remaining cases, 3 to $4 \mathrm{~L}$ of colon cleaning liquid was given in a polyethylene glycol-based preparation. All unexpected symptoms observed in the first 72 hours after the colonoscopy procedure, including pain, hemorrhage, peritoneal air collection, and fever, were recorded and analyzed as complications that were likely due to the colonoscopy.

\section{Statistics}

A normal distribution of data can be assumed in view of the amount of data available (about 750 observations from 600 patients). Continuous baseline descriptive variables were express- ed as means with standard deviations (SDs) and compared using a Student $t$-test. Categorical variables were expressed as absolute numbers and percentages. We used the $x^{2}$ statistic for most categorical variables, whereas the Fisher exact test was used for small numbers. Univariate and multivariate conditional logistic regression analyses were used to obtain crude and adjusted odds ratios (ORs) and 95\% confidence intervals (95\% Cls) after controlling simultaneously for potential confounders. Furthermore, $P$ values less than 0.05 were regarded as statistically significant, and those factors associated with mortality with a $P$ value of 0.1 or less were included in multivariate analysis using the Cox proportional hazards model. The survival curves were compared using the log-rank test and Cox models. The multivariate Cox models were also fitted to compare the cause of death in different situations and between groups to take into account potential confounders. All factors relating to organ failure (heart, liver, and kidney) and hospitalization in the ICU could be considered up-front confounders. A backward selection based on the $P$-value was carried out, including both categorical variables (heart failure, kidney failure, ischemic heart disease, valve replacement, auricular fibrillation, diabetes, stroke) as yes/no variables and continuous (survival) variables. The censure was done on death occurring before 30 days after the last urgent colonoscopy performed. Only hospitalization in the ICU was analyzed as a yes/no variable and as a stratification arm in the model variation. A two-sided $P$ value $<0.05$ was defined as significant. All analyses were performed using Systat version 6.1 .

\section{Results}

During the study period, 6,805 consecutive colonoscopies were performed in 6,600 patients. According to the criteria defined in the "Patients and Methods" section, 603 (9.13\%) patients had 757 (11\%) urgent colonoscopies. The mean number of colonoscopies per patient under this condition was 1.27 (range: $1-5$ per patient).

Baseline demographic and clinical characteristics of these patients are shown in $>$ Table 1 . The patients' mean age was 66.1 years (SD 15.6), and most were men (69\%). The majority had serious comorbidities, most of which were cardiovascular diseases, followed by digestive neoplasia and inflammatory or infectious diseases. The cardiovascular comorbidities consisted of ischemic heart disease (25\%), aorto-bifemoral vascular bypass $(9.6 \%)$, other vascular bypass $(15.6 \%)$, abdominal aortic aneurysm repair (15.4\%), heart transplant $(1.7 \%)$, heart failure (9.1\%), cardiac stent or bypass $(17.4 \%)$, and stroke $(8.1 \%)$. Moreover, $24 \%$ of patients had a history of neoplasia, including colorectal neoplasia ( $5 \%)$. Almost half of patients ( $n=295,49 \%)$ were hospitalized in the ICU due to septic shock, multivisceral failure, and hemorrhagic shock, which occurred in 15\%, $11 \%$, and $7 \%$ of patients, respectively ( $\triangleright$ Table 2 ). The remaining patients were hospitalized in other units (internal medicine, gastroenterology, surgery, geriatrics, etc.) in the hospital.

Among the 757 colonoscopies, nearly a third $(n=235,31 \%)$ allowed cecal intubation; in $71.2 \%$ of the cases $(n=539)$, bowel preparation was defined as "good" by the examiner, and in 
- Table 1 Baseline demographic and clinical characteristics of patients.

\begin{tabular}{|c|c|}
\hline \multicolumn{2}{|l|}{ Patients $(n=603)$} \\
\hline Age, mean (SD), years & $66.1(15.6)$ \\
\hline Sex, female/male, n & $187 / 416$ \\
\hline $\begin{array}{l}\text { Mean number (extremes) of colonoscopies per } \\
\text { patient }\end{array}$ & $1.27(1-5)$ \\
\hline \multicolumn{2}{|l|}{ Medical background } \\
\hline - Colorectal neoplasia & $5.1 \%(31)$ \\
\hline - Other neoplasia & $18.9 \%(114)$ \\
\hline - Inflammatory bowel disease & $3 \%(18)$ \\
\hline - Aorto-bifemoral vascular bypass & $9.6 \%(58)$ \\
\hline - Other vascular bypass & $15.6 \%(88)$ \\
\hline - Abdominal aortic aneurysm & $15.4 \%(93)$ \\
\hline - Ischemic heart disease & $25 \%(151)$ \\
\hline - Cardiac stent/bypass & $17.4 \%(105)$ \\
\hline - Heart transplant & $1.7 \%(10)$ \\
\hline - Valve replacement & $10.9 \%(66)$ \\
\hline - Heart failure & $9.1 \%(55)$ \\
\hline - Auricular fibrillation & $21.6 \%(130)$ \\
\hline - HBP & $47.8 \%(288)$ \\
\hline - Diabetes & $20.2 \%(122)$ \\
\hline - Chronic renal failure & $18.6 \%(112)$ \\
\hline - Dyslipidemia & $18.1 \%(109)$ \\
\hline - Stroke & $8.1 \%(49)$ \\
\hline Smoker & $21.6 \%(130)$ \\
\hline
\end{tabular}

- Table 2 Clinical outcomes.

\begin{tabular}{|l|c|}
\hline Patient hospitalized in ICU, number (\%) & $295(48.9 \%)$ \\
\hline - Multivisceral failure & $68(11.3 \%)$ \\
\hline - Hemorrhagic shock & $42(7 \%)$ \\
\hline - Cardiogenic shock & $55(9.1 \%)$ \\
\hline - Septic shock & $88(14.6 \%)$ \\
\hline Surgery & $67(11.1 \%)$ \\
\hline Mortality & $192(31.8 \%)$ \\
\hline
\end{tabular}

$61.8 \%$ of the cases $(n=468)$, colonoscopy was contributive according to the criteria defined above. Less than $10 \%$ of exams showed polyps $(n=46)$ or advanced colorectal neoplasia $(n=$ 20). No perforation was reported during or after the colonoscopies.

\section{Clinical symptoms and indications for colonoscopy and findings}

Gastrointestinal hemorrhage, reported in $36 \%$ of the cases ( $n=$ 214 ), was the main reason for urgent colonoscopy. In this context, 170 patients presented with rectal bleeding and 51 with melena (7 patients presented with both). All patients with melena underwent an upper $\mathrm{Gl}$ endoscopy prior to the colonoscopy that did not reveal the cause of the hemorrhage. Etiology of hemorrhage could be addressed during colonoscopy in $66.3 \%$ of the cases $(142 / 214)$. The main causes were diverticular bleeding ( $n=67,47.2 \%)$, ischemic colitis $(n=40,28.2 \%)$, tumoral lesions ( $n=11,7.7 \%)$, and angiodysplasia $(n=5,3.5 \%)$. Peri endoscopy therapeutic procedures were performed in 21 cases, as follows: 13 endoscopic clips with or without adrenalin serum injection, 2 band ligations, 4 argon-plasma coagulations (APCs), 1 adrenalin serum injection, and 1 bipolar coagulation. Radiological vascular embolization and urgent right hemicolectomy were necessary in 1 and 2 patients, respectively, because of peri endoscopic treatment failure. No fatal complication following colonoscopy was observed. Other colonoscopy indications were as follows: volvulus that needed urgent endoscopic management $(n=25)$, acute severe diarrhea $(n=44)$, or idiopathic acute intestinal pseudo-obstruction.

\section{Ischemic colitis}

The overall clinical and paraclinical features were suggestive of ischemic colitis before colonoscopy was performed in $44 \%$ of the cases $(n=264)$ because of clinical symptoms (hemorrhage, abdominal pain, organ failure), medical history (prior vascular surgery with abdominal aortic aneurysm repair or vascular bypass), or imaging (either computed tomography [CT] scan or magnetic resonance imaging [MRI] of abdomen) evidence. Among these patients, $77.6 \%(n=52)$ had a severe and necrotizing ischemic colitis and met the criteria of serious injury. The mean number of colonoscopies in these patients was 1.29 $(1-6)$ exams per patient.

\section{Patients undergoing surgery during the follow-up period}

There were 87 (40.6\%) patients with melena or rectal bleeding under monitoring in the ICU; among them, 67 patients $(11.1 \%$ of the cohort) required urgent surgery ( $\triangleright$ Table 2$)$. These patients were eligible for colectomy in emergency. Similarly, in 8 out of 25 patients with volvulus (11.9\% of whom underwent surgery), surgical sigmoidectomy was required. Other indications of urgent surgery were as follows: two right hemicolectomies for severe hemorrhage after the failure of endoscopic treatment; one segmental resection of the colon after postembolization perforation, which was indicated because of severe hemorrhage due to angiodysplasia; one colectomy for idiopathic acute intestinal pseudo-obstruction complicated by ischemic colitis; one colectomy for acute severe colitis due to ulcerative colitis; and two noncontributive exploratory laparotomy procedures without intestinal resection. 


\section{Early death as the main outcome}

Overall, 192 out of 603 patients died within 30 days after an urgent colonoscopy (mortality rate of $31.8 \%$ ), regardless of the number of colonoscopies $(1-6)$ performed per patient. As shown in $>$ Table 3 , a medical history of valve replacement $(P=$ $0.010)$, heart failure $(P=0.019)$, auricular fibrillation $(P=0.017)$, diabetes $(P=0.031)$, and chronic renal failure $(P=0.004)$ were significant predictors of mortality after univariate analysis. Similarly, diagnosis of ischemic colitis was a significant predictor of mortality $(P<0.001)$. In addition, hospitalization in the ICU $(P$ $<0.001)$, the presence of multivisceral failure $(P<0.001)$, hemorrhagic shock $(P=0.009)$, cardiogenic shock $(P<0.001)$, septic shock $(P<0.001)$, and the need for urgent surgery $(P=0.006)$ were significant predictors of mortality, whereas the presence of rectal bleeding $(P<0.001)$, diarrhea $(P=0.011)$, or volvulus $(P=0.020)$ was significantly protective. Multivariate analysis was then conducted, including 19 factors associated with 1month mortality and a p-value set at 0.1 or less, as observed in univariate analysis; this revealed five independent risk predictors of mortality ( $>$ Table 4$)$. Hospitalization in the ICU (whatever the reason), multivisceral failure, heart transplantation and ischemic colitis (as assessed by colonoscopy and confirmed by histology), and septic shock appeared to be indicators of bad prognosis. As indicated in - Fig. 1, the overall survival was better in patients without colonic ischemia. This difference was due to early mortality, as the difference did not persist in the later survival rate ( $>$ Fig. 1 ).

In this study, 128 patients underwent 300 urgent colonoscopies for ischemic colitis (2.3 exams per patient), which was staged as follows: stage 1: erythema and edema (28.1\% of the patients; $n=36$ ); stage 2 , non-necrotic ulcers (34.4\% of the patients; $n=44$ ); and stage 3 , extensive necrosis (37.5\% of the patients; $n=48)$. For nearly a third of these patients, ischemic colitis occurred after vascular surgery $(32.8 \%, n=42)$, and for $28.9 \%$ of the patients $(n=37)$, it occurred after an abdominal aortic aneurysm repair. The overall 1 -month mortality rate after colonoscopy in ischemic colitis patients reached $52 \%$ ( $n=$ $67)$.

In 52 of 128 ischemic colitis patients (40.6\%), urgent surgery was needed because of an uncontrolled clinical situation. Among these patients, 39 had early ( $<36$ hours after colonoscopy) and 13 had delayed surgery (after 36 hours or more). The overall mortality in these patients was $63 \%$ (33/52), and there was significant difference depending on whether the surgery was early (25 out of 39 ) or delayed (8 out of 13 ). Briefly, among those patients undergoing colon surgery, ischemic colitis appeared as a higher predictive condition of early mortality after surgery and rectorrhagia as the only predictive clinical symptom in the 13 cases of this condition included in the analysis.

No perforation was reported after 757 procedures. Moreover, in an analysis categorizing patients into two classes concerning cecal intubation - "Yes" versus "No" - with all new symptoms in the postcolonoscopy period considered as complications, there were no significant differences between those with complete colonoscopy ( $n=235$ out of 603 patients) undergoing the urgent procedure and the remaining cases.

\section{Discussion}

We took a pragmatic approached in this research and included all consecutive requests for colonoscopy in a large hospital over a 5-year study period. The benefits and outcomes of urgent bedside colonoscopy in consecutive patients, including not only those referred for bowel hemorrhage but also those with dramatic diarrhea of unknown cause of visceral failure, volvulus, and suspicion of ischemic colitis were reported. Furthermore, we investigated how this procedure could influence patients' early survival and showed that it is feasible, safe, and helpful for medical decision making. However, we also demonstrated that patients' morbidities can heavily affect the survival rate regardless of the indication or examination results ( $\triangleright \mathrm{Ta}$ ble 3).

This urgent procedure is presumed to enable diagnosis, such as by reaching site of the bleeding, allowing the intensity of the ischemia and its extensions to be evaluated, and allowing sampling of tissue and effluent [6-10]. This procedure may also offer important urgent therapeutic possibilities, such as stopping hemorrhage from diverticula or angiodysplasia or overpassing occlusion via devolvulation and stent use in volvulus and malignant tumor stenosis, respectively [10-16]. As far as we know, this is the largest cohort series based on 235 patients with cecal intubation; only 120-165 patients have been reported on in the other series [11-15]. Even in our series, the good preparation necessary for cecal intubation was the issue in one-third of all urgent procedures. Failure of preparation and difficulties in cecal intubation may enhance the rate of complications. Here, we also showed that this procedure is safe: Despite the failure of optimal preparation impeding cecal intubation, no severe complications, such as perforation, were observed in the 603 patients undergoing urgent procedures, regardless of whether the examination included complete cecal intubation. Nevertheless, the medical decisions and management based on colonoscopy results could be improved despite the incomplete colonoscopies evident in two-thirds of the cases. This means that urgent procedures could contribute to medical decisions and management even when there is insufficient preparation and failure of cecal intubation. More difficulties related to cecal intubation were also observed in patients with one or more comorbidities. Among the symptoms and conditions leading to urgent colonoscopy, even in those patients hospitalized in the $\mathrm{ICU}$, red bowel bleeding, diarrhea, and colonic volvulus appeared to be protective, as they revealed causes like diverticular hemorrhage, IBD or infection, and volvulus. The main cause of hemorrhage was diverticula in our series, followed by ischemia and tumors, as in several other series $(3,16-17)$.

Abdominal imaging was performed in all cases hospitalized in ICU prior to the colonoscopy; this was helpful for diagnosis, as previously reported [18]. The peri endoscopic therapeutic procedures were successful in $19.5 \%$ of cases for hemorrhage, which is close to the proportion of $25.6 \%$ reported by Nagata et al. [15]. However, worse outcomes were observed in those pa- 
Table 3 Univariate analysis of risk factors of death.

\begin{tabular}{|c|c|c|c|}
\hline & Alive $(n=411)$ & Deceased $(n=192)$ & $P$ \\
\hline Age, mean (SD), years & $64.2(0.84)$ & $66.6(0.95)$ & 0.074 \\
\hline Sex, female/male, n & $133 / 278$ & $54 / 138$ & 0.3 \\
\hline Smoker & $91(22.1 \%)$ & $39(20.3 \%)$ & 0.346 \\
\hline \multicolumn{4}{|l|}{ Medical background } \\
\hline - Colorectal neoplasia & $21(6.4)$ & $10(3.7)$ & 0.135 \\
\hline - Other neoplasia & $75(18.2 \%)$ & $39(20.3 \%)$ & 0.309 \\
\hline - Inflammatory bowel disease & $15(3.6 \%)$ & $3(1.6 \%)$ & 0.318 \\
\hline - Aorto-bifemoral bypass & $41(10 \%)$ & $17(4.1 \%)$ & 0.392 \\
\hline - Other vascular bypass & $60(14.6 \%)$ & $28(6.8 \%)$ & 0.551 \\
\hline - Abdominal aortic aneurysm & $63(15.3 \%)$ & $30(15.6 \%)$ & 0.507 \\
\hline - Ischemic heart disease & $97(23.6 \%)$ & $54(28.1 \%)$ & 0.137 \\
\hline - Cardiac stent/bypass & $68(16.5 \%)$ & $37(19.3 \%)$ & 0.238 \\
\hline - Heart transplant & $4(1 \%)$ & $6(3.1 \%)$ & 0.061 \\
\hline - Valve replacement & $36(8.7 \%)$ & $30(15.6 \%)$ & 0.010 \\
\hline - Heart failure & $30(7.3 \%)$ & $25(13 \%)$ & 0.019 \\
\hline - Auricular fibrillation & $78(19 \%)$ & $52(27.1 \%)$ & 0.017 \\
\hline - HBP & $193(47 \%)$ & $95(49.5 \%)$ & 0.312 \\
\hline - Diabetes & $74(18 \%)$ & $48(25 \%)$ & 0.031 \\
\hline - Chronic renal failure & $64(15.6 \%)$ & $48(25 \%)$ & 0.004 \\
\hline - Dyslipidemia & $75(18.2 \%)$ & $34(17.7 \%)$ & 0.485 \\
\hline - Stroke & $28(6.8 \%)$ & $21(5.1 \%)$ & 0.061 \\
\hline \multicolumn{4}{|l|}{ Clinical symptoms } \\
\hline - Rectal Bleeding & $135(32.8 \%)$ & $35(18.2 \%)$ & $<0.001$ \\
\hline - Melena & $39(9.5 \%)$ & $12(6.2 \%)$ & 0.119 \\
\hline - Diarrhea & $37(9 \%)$ & $7(3.6 \%)$ & 0.011 \\
\hline \multicolumn{4}{|l|}{ Diagnosis } \\
\hline - Volvulus & $22(5.4 \%)$ & $3(1.6 \%)$ & 0.020 \\
\hline - Ischemic colitis & $61(15 \%)$ & $67(35 \%)$ & $<0.001$ \\
\hline \multicolumn{4}{|l|}{ Clinical outcomes } \\
\hline Hospitalization in ICU & $156(38 \%)$ & $137(71.3 \%)$ & $<0.001$ \\
\hline - Multi organ Failure & $21(5.1 \%)$ & $47(24.5 \%)$ & $<0.001$ \\
\hline - Hemorrhagic shock & $21(5.1 \%)$ & $21(11 \%)$ & 0.009 \\
\hline - Cardiogenic shock & $23(5.6 \%)$ & $32(16.7 \%)$ & $<0.001$ \\
\hline - Septic shock & $42(10.2 \%)$ & $46(11.2 \%)$ & $<0.001$ \\
\hline Surgery & $36(8.8 \%)$ & $31(16.1 \%)$ & 0.006 \\
\hline
\end{tabular}



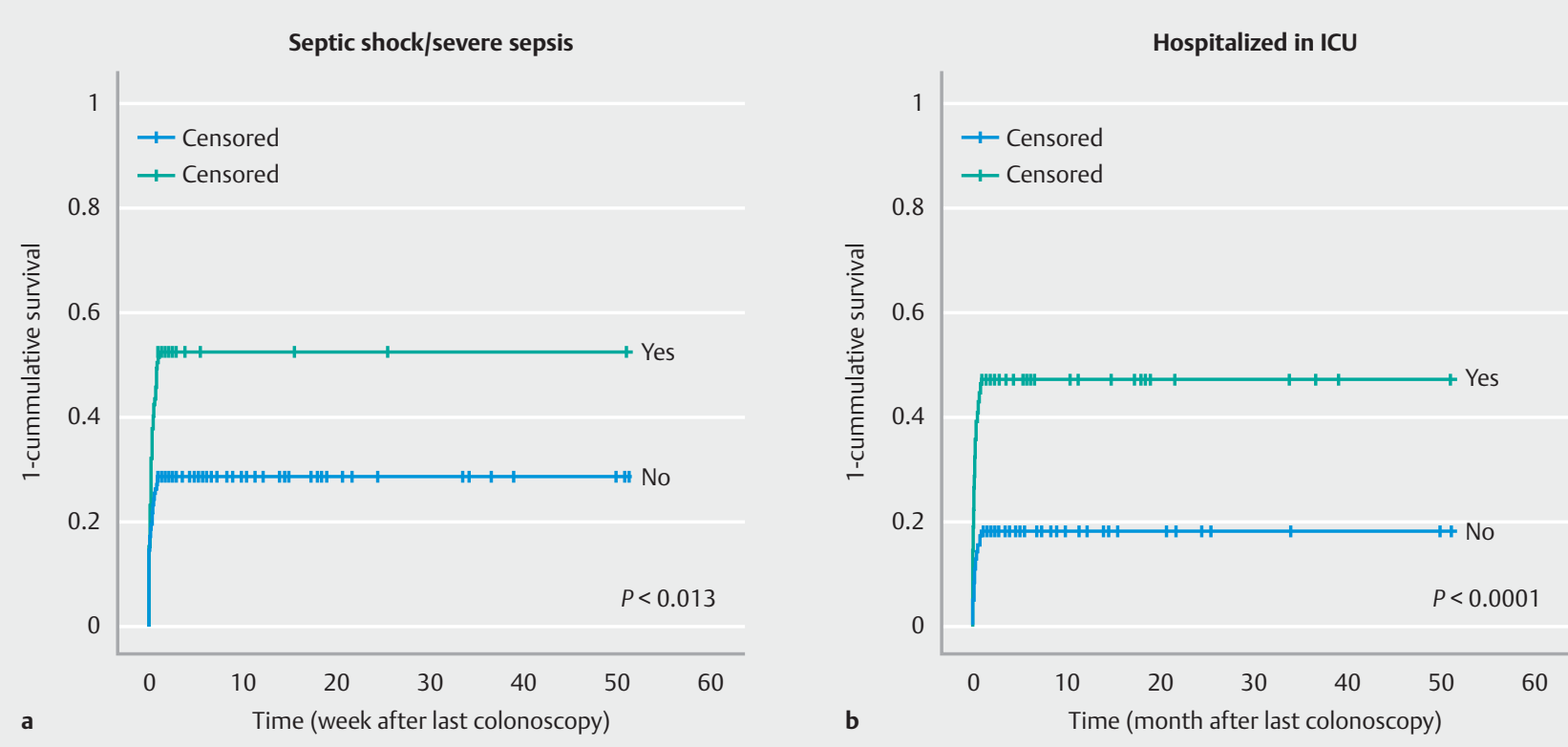

Shock/multi organ failure

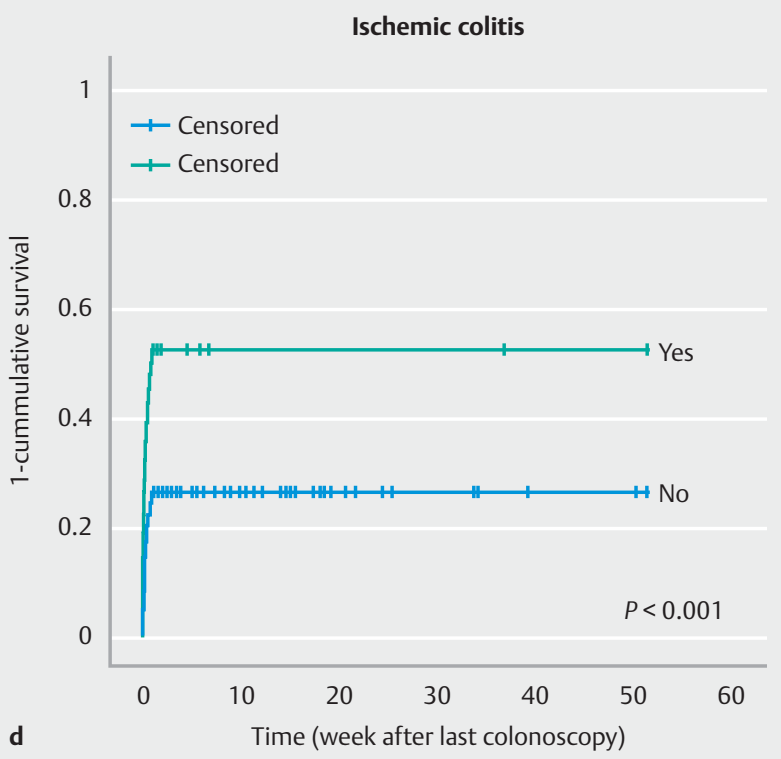

Fig. 1 Probability of survival in patients undergoing emergency colonoscopy.

tients with ischemic colitis, in whom the highest rate of early mortality was found.

Colonic ischemia, as assessed by colonoscopy and pathology, appeared to be one of the main independent risk factors of mortality following heart transplant, multivisceral failure, and severe sepsis. This means that physicians should take into consideration the feasibility risk - benefit analysis of the procedure, especially in patients with comorbidities. The mechanism of ischemia was cardiovascular failure in most cases, and this could lead to early mortality when associated with multivisceral failure or septic shock. This finding was in accordance with previous studies [19-22]. We speculate that those patients with ischemic colitis and comorbidities, such as visceral failure or septic shock, who underwent colon surgery experienced bad
- Table 4 Multivariate analysis of risk factors of death.

\begin{tabular}{|l|l|l|l|}
\hline & Odds Ratio & IC 95\% & $\boldsymbol{P}$ \\
\hline Multi-organ failure & 21.5 & $(19.3-23.6)$ & $<\mathbf{0 . 0 0 1}$ \\
\hline Hospitalization in ICU & 20.3 & $(18-55.5)$ & $<\mathbf{0 . 0 0 1}$ \\
\hline Ischemic colitis & 13.9 & $(12.4-14.5)$ & $<\mathbf{0 . 0 0 1}$ \\
\hline Heart transplant & 9.37 & $(8.11-11.71)$ & $<\mathbf{0 . 0 1}$ \\
\hline Septic shock & 6.2 & $(4.7-7.8)$ & $\mathbf{0 . 0 1 3}$ \\
\hline
\end{tabular}


outcomes because in these situations the, benefit of urgent colonoscopy - regardless of whether colonic surgery was required - appears questionable due to the low benefit of colon surgery afterward. By contrast, those patients with acute colonic distension or acute diarrhea who were hospitalized in the ICU ( $10 \%$ of cases) without visceral failure experienced better outcomes, since they survived for more than 1 month after the colonoscopy date.

Our study had several limitations. First, this was a retrospective study with potential recall bias. Second, in general, medical decisions - particularly those regarding urgent surgery should be enrolled from the same source population or decision chart with pre-established criteria from which the cases were selected for surgery. In addition, a bias due to the screening code for selecting patient here was linked with colonoscopy examination; this may have influenced the current results, as we could not include those patients for whom the procedure was requested but not performed due to death occurring before the examination. Finally, we cannot rule out the bias in medical decisions by default or in excess due to the period this study was conducted; however, it appears unlikely that such bias was present, as there was no significant change in the clinical pathway and management, physician experience, or surgery decision-making process observed in the units of gastroenterology, surgery, or the ICU during the study period. Despite these limitations, we report the largest retrospective series in the literature on the feasibility and contribution of urgent colonoscopy in routine health care [5-10].

\section{Conclusion}

The present study indicated that the current decision tree for urgent colonoscopy should include comorbidities to evaluate the risks and benefits of the procedure, as well as time for its release. The most critical situation concerns colonic ischemia with comorbidities that could impact the outcome, whether cecal intubation can be performed during the procedure, and whether diagnosis is based on a single or repeated urgent colonoscopy procedure.

\section{Acknowledgements}

The authors thank the Association Charles Debray for financial support and editing fees for the study.

\section{Competing interests}

EA received support from Fujinin Company for accommodations for the congress. MF received support from IPSEN for accommodations for the congress. AA has received consulting fees from AbbVie, Takeda, Gilead and Biocodex as well as lecture fees and travel accommodations from AbbVie, Biocodex, Ferring, Takeda, and MSD, and advisory board fees from Gilea, Takeda, and AbbVie. LY has received support from Olympus, Mayoli and Fujinon for accommodations for the congress. SI has received support for travel accommodations from Pfizer, Novartis, Biocodex, and IPSEN.

References

[1] Green BT, Rockey DC, Portwood G et al. Urgent colonoscopy for evaluation and management of acute lower gastrointestinal hemorrhage: a randomized controlled trial. Am J Gastroenterol 2005; 100: 23952402

[2] Sengupta N, Tapper EB, Feuerstein JD. Early Versus Delayed Colonoscopy in Hospitalized Patients With Lower Gastrointestinal Bleeding: A Meta-Analysis. J Clin Gastroenterol 2016; 51: 352 - 359

[3] Jensen DM, Machicado GA, Jutabha R et al. Urgent Colonoscopy for the Diagnosis and Treatment of Severe Diverticular Hemorrhage. N Engl J Med 2000; 342: 78-82

[4] Lanas A, Garcia-Rodriguez LA, Polo-Tomas M et al. Time trends and impact of upper and lower gastrointestinal bleeding and perforation in clinical practice. Am J Gastroenterol 2009; 104: 1633 - 1641

[5] Longstreth GF. Epidemiology and outcome of patients hospitalized with acute lower gastrointestinal hemorrhage: a population-based study. Am J Gastroenterol 1997; 92: 419-424

[6] Church J, Kao J. Bedside colonoscopy in intensive care units: indications, techniques, and outcomes. Surg Endosc 2014; 28: 2679-2682

[7] Wolff JH, Rubin A, Potter JD et al. Clinical significance of colonoscopic findings associated with colonic thickening on computed tomography: is colonoscopy warranted when thickening is detected? J Clin Gastroenterol 2008; 42: 472-475

[8] Kim BC, Cheon JH, Kim TI et al. Risk factors and the role of bedside colonoscopy for lower gastrointestinal hemorrhage in critically ill patients. Hepatogastroenterology 2008; 55: 2108-2111

[9] Horiuchi A, Nakayama Y. Urgent Colonoscopy for the Diagnosis and Treatment of Severe Lower GI Bleeding and Acute Colorectal Obstruction. Gastrointest Endosc 2005; 61: 201-206

[10] García SánchezM, González GalileaA, LópezVallejos P et al. Role of early colonoscopy in severe acute lower gastrointestinal bleeding. Gastroenterol Hepatol 2001; 24: 327-332

[11] Jensen DM, Machicado GA, Jutabha R et al. Urgent colonoscopy for the diagnosis and treatment of severe diverticular hemorrhage. N Engl J Med 2000; 342: $78-82$

[12] Nagata N, Niikura R, Aoki T et al. Risk factors for adverse in-hospital outcomes in acute colonic diverticular hemorrhage. World J Gastroenterol 2015; 21: 10697-10703

[13] Niikura R, Nagata N, Shimbo T et al. Adverse Events during Bowel Preparation and Colonoscopy in Patients with Acute Lower Gastrointestinal Bleeding Compared with Elective Non-Gastrointestinal Bleeding. PLoS One 2015; 10: doi:10:e0138000.doi

[14] Sugihara Y, Kudo SE, Miyachi H et al. Analysis of Risk Factors for Colonic Diverticular Bleeding: A Matched Case-Control Study. Gut Liver 2016; 10: $244-249$

[15] Nagata N, Niikura R, Sakurai T et al. Safety and Effectiveness of Early Colonoscopy in Management of Acute Lower Gastrointestinal Bleeding on the Basis of Propensity Score Matching Analysis. Clin Gastroenterol Hepatol 2016; 14: 558-564

[16] Green BT, Rockey DC, Portwood G et al. Urgent Colonoscopy for Evaluation and Management of Acute Lower Gastrointestinal Hemorrhage: A Randomized Controlled Trial. Am J Gastroenterol 2005; 100: $2395-2402$

[17] Navaneethan U, Njei B, Venkatesh PG et al. Timing of colonoscopy and outcomes in patients with lower Gl bleeding: a nationwide population-based study. Gastrointest Endosc 2014; 79: 297-306 
[18] Nagata N, Niikura R, Aoki T et al. Role of urgent contrast-enhanced multidetector computed tomography for acute lower gastrointestinal bleeding in patients undergoing early colonoscopy. J Gastroenterol 2015; 50: $1162-1172$

[19] Becquemin JP, Majewski M, Fermani N et al. Colon ischemia following abdominal aortic aneurysm repair in the era of endovascular abdominal aortic repair. J Vasc Surg 2008; 47: $258-263$
[20] Huguier M, Barrier A, Boelle PY et al. Ischemic colitis. Am J Surg 2006; 192: $679-684$

[21] Chavalitdhamrong D, Jensen DM, Kovacs TO et al. Ischemic colitis as a cause of severe hematochezia: risk factors and outcomes compared with other colon diagnoses. Gastrointest Endosc 2011; 74: 852 - 857

[22] Reissfelder C, Sweiti H, Antolovic D et al. Ischemic colitis: Who will survive? Surgery 2011; 149: 585-592 